\title{
EVALUATION OF TUNNEL EXCAVATION COMBINING TERRESTRIAL LASER SCANNING POINT CLOUDS AND DESIGN MODELS
}

\author{
Yuzhou Zhou ${ }^{1}$, Zhen Dong ${ }^{1}$, Peiling Tong ${ }^{2}$, Bisheng Yang ${ }^{1 *}$ \\ ${ }^{1}$ State Key Laboratory of Information Engineering in Surveying, Mapping and Remote Sensing, Wuhan University, \\ Wuhan 430079, China - (zhouyuzhou, dongzhenwhu, bshyang)@ whu.edu.cn \\ ${ }^{2}$ School of Resource and Environmental Sciences, Wuhan University, \\ Wuhan 430079, China - (tongpeiling)@ whu.edu.cn
}

\section{Commission II, WG II/3}

KEY WORDS: Terrestrial Laser Scanning, Tunnel Excavation Evaluation, Cross-Section, Design Model, Three-Dimensional Reconstruction, Centerline Fitting.

\begin{abstract}
:
The quality of tunnel excavation is evaluated by comparing the excavated tunnel and the design model. Terrestrial laser scanning (TLS) provides surveyors with dense and accurate three-dimensional (3D) point clouds for excavation model reconstruction. However, sufficient attention has not been paid to incorporating design models for tunnel point cloud processing. In this paper, a technical framework that combines TLS point clouds and the design model for tunnel excavation evaluation is proposed. Firstly, the point clouds are sliced into cross-sections and the feature points are accordingly extracted. Then, considering the structure of the design model, feature point deficiencies are repaired by topological and parametric model interpolation. Finally, the excavation quality is evaluated in terms of the deviation of centerlines and 3D models. This method is validated in the case study. Experiments show that the deviation of centerline azimuth is acceptable but there remain considerable overbreak and underbreak, which respectively account for $20.6 \%$ and $11.2 \%$ of the design excavation volume.
\end{abstract}

\section{INTRODUCTION}

As important infrastructures of water conservancy and transportation engineering, a considerable number of tunnels have been excavated in recent years. Design models (Figure 1 (a) (b)) are primary guidelines for tunnel excavation. During the excavation by drilling or blasting, constructors have to evaluate the deviation between the excavated tunnel and the design model, including the volume of underbreak or overbreak and the bias of centerlines (Cui et al., 2019; Fekete et al., 2010). These indicators not only reflect the quality of excavation but also guide the subsequent project arrangement (Xu et al., 2018). Besides, design models provide an important basis for tunnel point clouds analysis as a local position reference.

In the past decade, terrestrial laser scanning (TLS) has been widely used in the feature extraction and three-dimensional (3D) reconstruction of tunnel excavation (Figure 1 (c)), since it can efficiently collect dense and accurate $3 \mathrm{D}$ point clouds in the tunnel (Dong et al., 2020; Panella et al., 2020). Some early studies revealed the potentials and challenges of laser scanning for tunnel construction management (Fekete et al., 2010; Wang et al., 2014). Pejić (2013) discussed the general principles for planning and optimizing the deployment of laser scanning towards better point completeness and measurement accuracy. When processing the collected data, it is a straightforward idea to create a polygon mesh from the point clouds, which offers the advantage of convenient volume calculation and intuitive visualization. However, case studies demonstrated that creating a 3D polygon mesh model was demanding in terms of computational resources, and led to geometry ambiguity in the form of overlapping faces (Gikas, 2012).

\footnotetext{
* Corresponding author
}
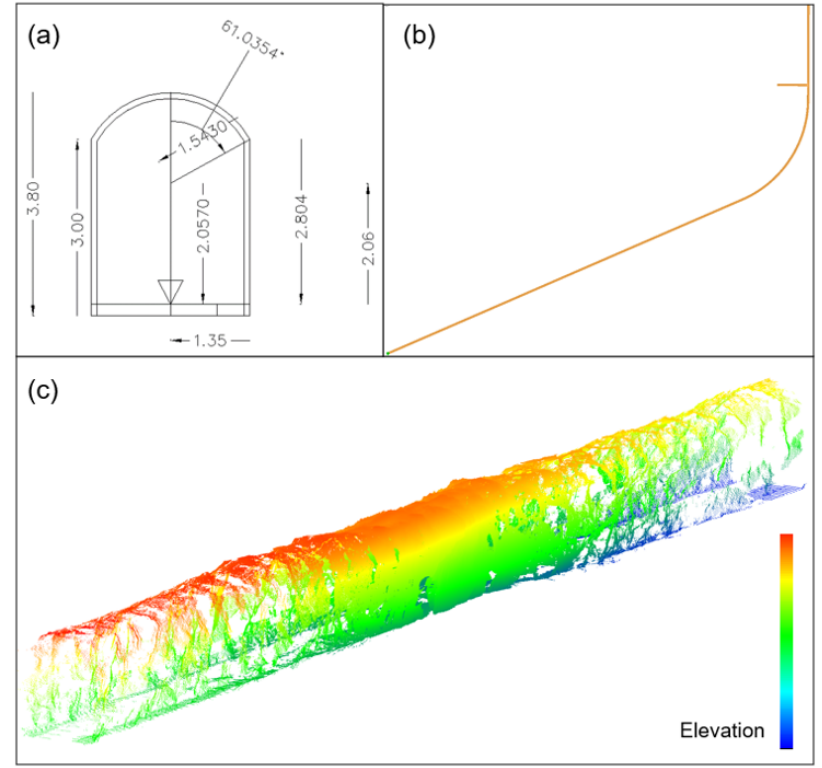

Figure 1. The design model and terrestrial laser scanning point clouds of a tunnel. (a) Design cross-section; (b) design 3D solid model; (c) terrestrial laser scanning point clouds after excavation.

Thereby, extra labor is required to improve the quality of the 3D model. Recent studies generally segment the point clouds into cross-sections for further analysis and 3D model creation, which conforms to the convention of designers and constructors (Attard et al., 2018; Yi et al., 2019). Kang et al. (2014) extracted the tunnel central axis using a segment-wise fitting strategy and proposed an interpolation algorithm to enhance the 
cross-section point clouds. Qiu and Cheng (2017) also extracted cross-section points based on central axis calculation, and further removed the non-lining points for the DEM (Digital Elevation Model) generation. To reduce the interference of cables and other equipment in tunnels, Xu et al. (2018) adopted iterative circular fitting, and Cui et al. (2019) used wavelet filtering. However, there are still several open problems.

Great progress has been made in applications of terrestrial laser scanning for tunnels. First, TLS suffers inconsistency of point density and deficiency by rock occlusion, challenging the 3D model reconstruction (Soudarissanane et al., 2011). Second, tunnel point clouds are generally processed without considering the structure of the design model. Third, the volume and position of rock overbreak or underbreak should be calculated and intuitively demonstrated.

Therefore, in this work, the evaluation of tunnel excavation is carried out by integrating TLS point clouds and the design model. The main contributions are as follows.

- A set of feature points are extracted to delineate the contour of each cross-section. Then the feature point deficiencies that correspond to different structures of the design model are completed separately. These feature points constitute the contour of the excavation and facilitate subsequent $3 \mathrm{D}$ evaluation.

- The centerline of the excavation is reconstructed according to the design alignments by minimizing a customized residual function. Meanwhile, the volume and position of rock overbreak and underbreak are evaluated based on the reconstructed excavation model and design model.

\section{METHOD}

The proposed framework mainly consists of three steps. First, after the data preprocessing, point clouds are sliced and projected into cross-sections to extract feature points. Second, topological and parametric models are generated to complete the feature point deficiencies. Third, incorporating the design model, the excavation quality is evaluated in terms of centerlines and $3 \mathrm{D}$ models. Figure 2 demonstrates the pipeline of the proposed method.

\subsection{Cross-Section Feature Point Extraction}

Point clouds are firstly registered to the local construction coordinate system where the model is designed. The noise and points below ground reflected by water are then removed. Subsequently, the data are subsampled by assigning a minimum distance between points to reduce data redundancy. The centerline of the design model is the reference for the cross-section slicing. For each cross-section, the points are split into different groups according to their relative positions to the design model. Specifically, the profile of the design cross-section $S$ is firstly split into segments $S_{i=1 \cdots n}$. If the shortest line link from point $P$ to $S$ falls on $S_{i}$, then $P$ is assigned to point group $G_{i}$. Next, the outermost point relative to the design model in $G_{i=1 \cdots n}$ is selected as the representative feature point. These feature points delineate the contour of cross-sections and in the meantime eliminate the interference of in-tunnel object points (e.g., people, survey instruments). Figure 3 illustrates the point groups and the extracted contour.

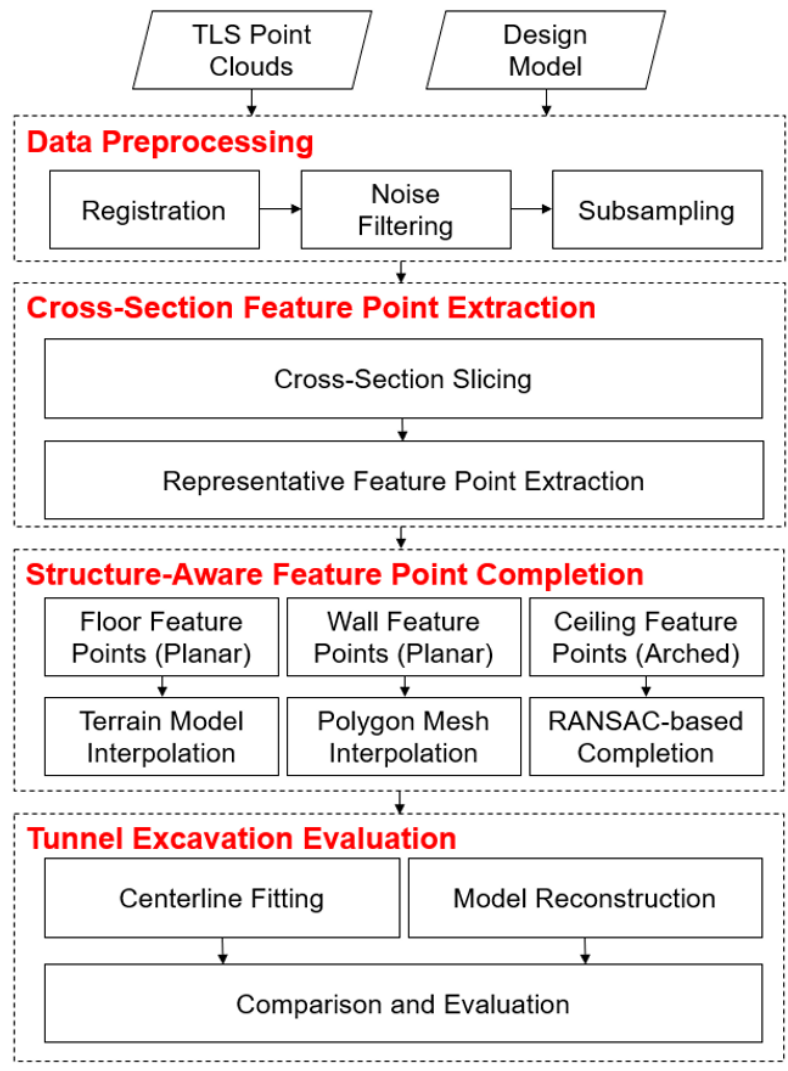

Figure 2. Overview of the proposed framework.

\subsection{Structure-Aware Feature Point Completion}

Considering point deficiencies between stations caused by occlusion, it is beneficial to generate a complete and evenly distributed point set towards an accurate tunnel 3D model. After the feature point extraction, if point group $G_{i}$ is empty, the segment $S_{i}$ of this cross-section is considered suffering missing points. In this step, both the previously extracted feature points and the structure of the design model are considered. It is assumed that the neighboring feature points and the design model jointly introduce the local geometric characteristics of point deficiencies. The feature points are classified into floor points, lateral wall points and ceiling points because the models are designed by integrating various geometric elements. For example, the tunnel used in our experiment features an arched ceiling but a planar wall. Concretely, floor feature points support the construction of an in-tunnel topographic terrain model, so that the tunnel floor is completed by interpolation of the model. Similarly, feature points corresponding to lateral walls are connected to a polygon mesh for completion. As for point completion on the arched ceiling, a local parametric model is fitted after inlier extraction via RANSAC (RANdom SAmple Consensus) (Nurunnabi et al., 2017; Schnabel et al., 2007). The structure of the design model is involved for the selection of geometric models when building connections of feature points.

\subsection{Tunnel Excavation Evaluation}

Centerline Fitting and Evaluation Lines, arcs and spirals constitute the horizontal curve of the design model. Mathematically, they are represented by a common parametric model in Equation (1) but in different parameter ranges (Table 1). The curve length parameter $u$ denotes the $2 \mathrm{D}$ curve length from 


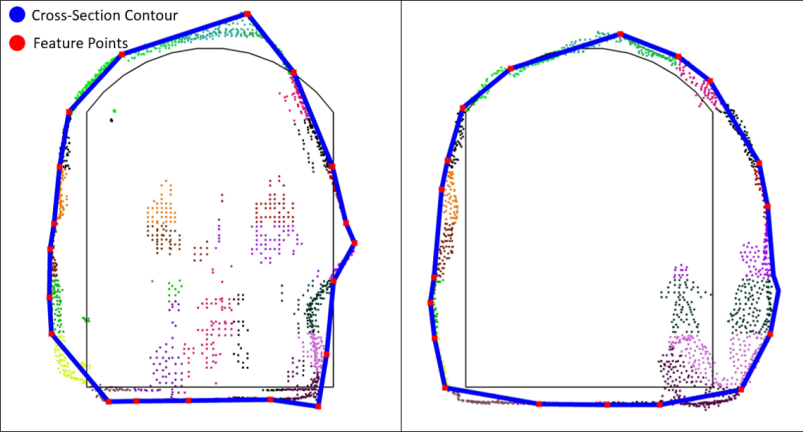

Figure 3. Cross-section point segmentation and the extracted feature points and contours. The point groups $G_{i=1 \cdots n}$ in each cross-section is demonstrated in different colors. The red points are the extracted feature points. The blue lines are the contours.

The black curve is the profile of the design cross-section $S$.

$\left(X_{0}, Y_{0}\right)$ to $(X(u, p), Y(u, p))$ and $p$ denotes the curve parameters $\{\mu, \kappa, \psi\}$.

$$
\left\{\begin{array}{l}
X(u, p)=X_{0}+\int_{0}^{u} \cos \left(\mu+\kappa t+\frac{1}{2} \psi t^{2}\right) d t \\
Y(u, p)=Y_{0}+\int_{0}^{u} \sin \left(\mu+\kappa t+\frac{1}{2} \psi t^{2}\right) d t
\end{array}\right.
$$

\begin{tabular}{cccc}
\hline Curve Element & $\mu$ & $\kappa$ & $\psi$ \\
\hline Lines & {$[0,2 \pi)$} & 0 & 0 \\
Arcs & {$[0,2 \pi)$} & {$[-0.05,0.05]$} & 0 \\
Spirals & {$[0,2 \pi)$} & {$[-0.05,0.05]$} & {$[-0.0025,0.0025]$} \\
\hline
\end{tabular}

Table 1. Parameter ranges for horizontal design curve elements.

The centroid of the completed feature points $c_{i=1 \cdots n_{c}}$ in each cross-section is extracted to be fitted. First, $c_{i=1 \cdots n_{c}}$ is segmented based on the design centerline into $n_{s}$ segments. For instance, $n_{s}=3$ in our experiment data, and the centroid points which fall in the circular arc segments will be fitted according to the parameter range of arcs in Table 1. The location of $\left(X_{0}, Y_{0}\right)$, as the starting point of each segment, is thus determined by the segmentation result. Then, global curve fitting is performed by minimizing a customized residual function $R$ as shown in Equation (2), where $\boldsymbol{P}$ denotes the curve parameter $p^{j=1 \cdots n_{s}}$ for each curve segment. $n_{c}$ denotes the number of centroid points and $n_{s}$ denotes the number of design curve segments.

$$
R=\sum_{i=1}^{n_{c}} D_{i}(\boldsymbol{c}, \boldsymbol{P})+\sum_{j=1}^{n_{s}-1} \lambda \Delta_{(j, j+1)}
$$

In Equation (2), $D_{i}(\boldsymbol{c}, \boldsymbol{P})$ denotes the residual from the centroids $c$ and the parametric curve segments, whose parameter is $p_{j} \in \boldsymbol{P}$, as shown in Equation (3). This term is based on the primary principle that the fitted curve should be close to the extracted centroid points.

$$
D_{i}(\boldsymbol{c}, \boldsymbol{P})=\left\|\left(X_{i}, Y_{i}\right)-\left(X\left(u_{i}, p^{j}\right), Y\left(u_{i}, p^{j}\right)\right)\right\|
$$

Besides, to encourage the fitting method to fill the spatial gaps on segment junctions, we introduce the smooth terms $\Delta_{(j, j+1)}$. $\Delta_{(j, j+1)}$ denotes the intersection gap between the $p$ th and the $p+1$ th curve segments as shown in (4), where $l^{j}$ is the curve length of the $j$ th segment.

$$
\Delta_{(j, j+1)}=\left\|\left(X_{0}^{j+1}, Y_{0}^{j+1}\right)-\left(X\left(l^{j}, p^{j}\right), Y\left(l^{j}, p^{j}\right)\right)\right\|
$$

After the centroid points segmentation, the residual function in Equation (2) is globally formulated and minimized for the tunnel. This residual function takes different curve elements into consideration and helps generate a segment-wise curve that shares consistent curve models with the design model. Also, the continuity between segments is guaranteed due to the smooth term. This facilitates the parametric evaluation of centerlines comparing to the design model.

Model Reconstruction and Comparison Based on the feature points after completion, the 3D excavation mesh model is constructed and contrasted to the design model as shown in Figure 4 . Because the feature points are selected or complemented as representative points from each cross-section, directly constructing a polygon mesh will not lead to geometry confusion like face overlapping. Boolean operations are performed for the comparison between the excavation model and the design model. Their intersection shows the volume of valid excavation. Meanwhile, the overbreak and underbreak models are constructed by $3 \mathrm{D}$ mesh model subtraction.

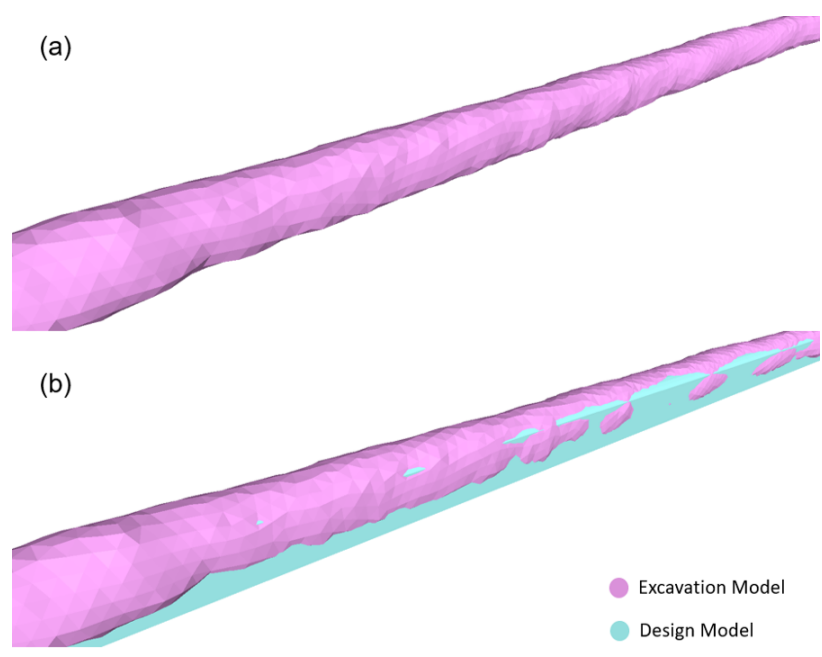

Figure 4. The reconstructed excavation model and the design model. (a) The reconstructed 3D excavation model; (b) the excavation model overlaid on the design model.

\section{RESULTS AND DISCUSSION}

Dataset Overview The terrestrial laser scanning data was collected during the extension project of Wuqiang Creek Hydropower Station, Hunan Province, China. The scanning equipment was Leica ScanStation P40 and the point clouds were registered using the preset tie points. Additionally, the design model, including a measurable entity model and the parameters for cross-sections and centerlines, was provided by the tunnel designers. The designed tunnel centerline contains straight lines and arcs, of which the total length is about $820 \mathrm{~m}$. The designed cross-section features an arched ceiling. Figure 1 demonstrates the data for experiments. 
Parameter Settings In our experiment, the inter-slice distance between the successive cross-sections is set to $0.5 \mathrm{~m}$. Experiments showed that the inter-slice distances ranging from 0.2 $\mathrm{m}$ to $1.0 \mathrm{~m}$ were all feasible in this dataset. Closer inter-slice distance leads to more missing points in cross-sections, and a larger value causes the loss of small geometry features. Each cross-section was segmented into 20 parts when extracting feature points, which was tuned empirically. When performing structure-aware feature point completion, the neighboring region of a cross-section was set to $2.5 \mathrm{~m}$ along the centerline.

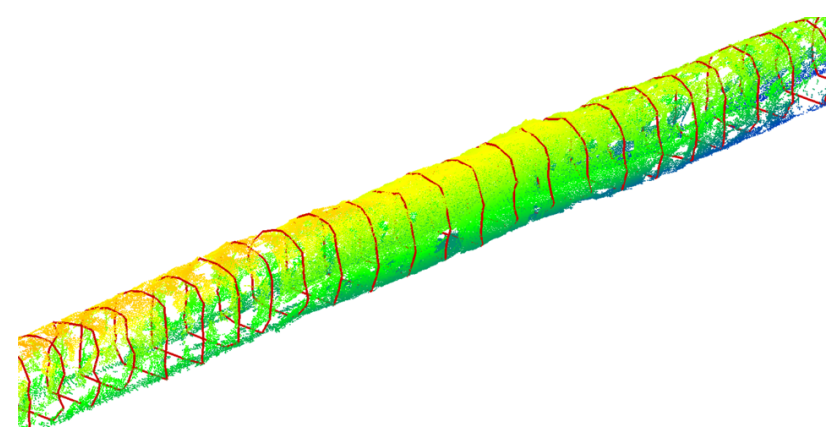

Figure 5. The extracted tunnel cross-sections overlaid on the point clouds. Only the first one of each five successive cross-sections is shown (i.e., $2.5 \mathrm{~m}$ interval).

Qualitative Results The initial cross-section feature points were refined with the structure-aware feature point completion so that they can outline the tunnel profiles even with point deficiencies between scanning stations, as shown in Figure 5. The centerline of excavation was extracted as parametric curve segments as shown in Figure 6, which is in accordance with the design specifications. The completed tunnel grid DEM (Digital Elevation Model) was also interpolated and rendered based on the floor feature points. Accordingly, parametric curve evaluation was performed in terms of the bias of azimuth and curvature radius. In Figure 7, the comparison between the tunnel excavation model and the design model based on model boolean operations is demonstrated. Although the excavation was according to the design model, there remained evident overbreak and underbreak, which were rendered and measured in the result.

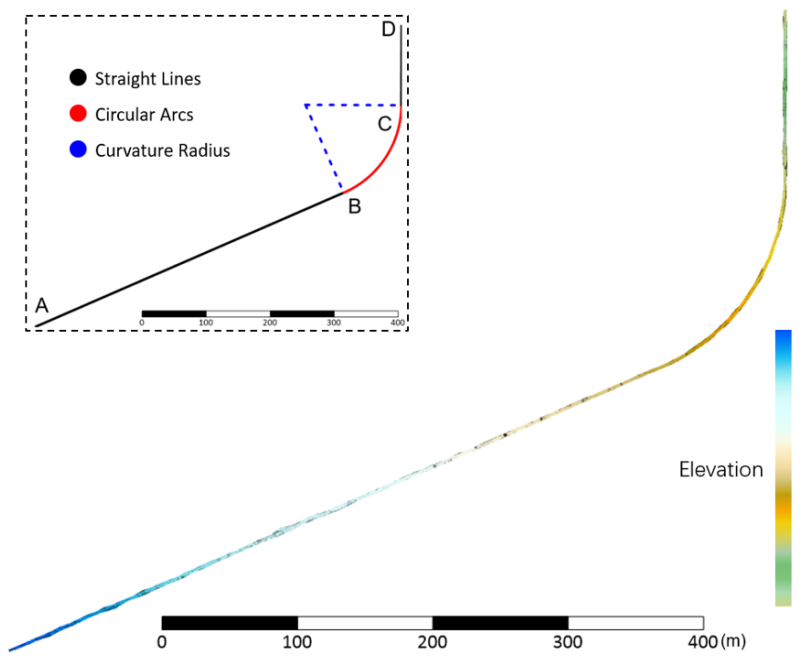

Figure 6. The reconstructed terrain model and the extracted centerline curve elements of the excavation tunnel.

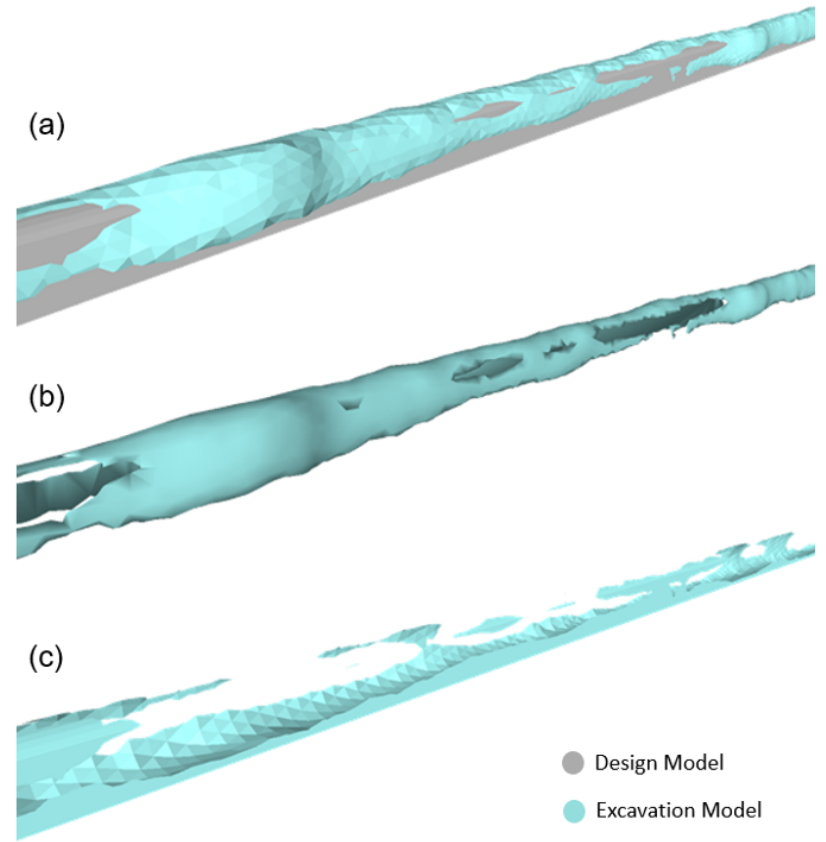

Figure 7. Excavation model evaluation. (a) The excavation model and the design model; (b) the overbreak model; (c) the underbreak model.

Quantitative Results Segment-wise centerline deviation evaluation was performed according to the extracted parameters and the design parameters. According to the experiment result shown in Table 2, the azimuth bias of segment $\mathrm{AB}$ is $0.017^{\circ}$ and the azimuth bias of segment $\mathrm{CD}$ is $0.002^{\circ}$. The curvature radius of the design model of segment $\mathrm{BC}$ is $150.00 \mathrm{~m}$ and the extracted curvature radius is $149.951 \mathrm{~m}$. These results reveal that the bias between the excavation centerline and the design centerline is acceptable for constructors. However, it is noticed that there remains the bias of starting points for each segment of the excavated centerline, which is caused by the evident overbreak or underbreak.

The excavation model was reconstructed and evaluated according to the design model. The overbreak and underbreak models were constructed as shown in Figure 7. Experiment results showed that the overbreak volume is $1730.9 \mathrm{~m}^{3}$ and the underbreak volume is $943.1 \mathrm{~m}^{3}$, which respectively account for $20.6 \%$ and $11.2 \%$ of the design excavation volume.

Analysis of Feature Point Completion The structure-aware point cloud completion was shown valid for increasing the consistency between excavation and the reconstructed 3D model as shown in Figure 8. Figure 8 (a) illustrates that the point clouds suffered considerable deficiencies, making the reconstructed 3D model failed to outline the tunnel (Figure 8 (c)). Without the completion, the point clouds in certain regions were too sparse to provide enough geometric characteristics for 3D reconstruction. The completion strategy integrates the structure of the design model (Figure 8 (b)) and the characteristics of local point clouds, and thus provides better 3D modeling performance (Figure 8 (d)).

Limitations and Outlooks The inter-slice distance between cross-sections and the number of feature points in each crosssection determine the level of geometric details. However, they are tuned empirically in the proposed method. In recent years, mobile tunnel inspecting systems have shown great potential 


\begin{tabular}{|c|c|c|c|c|c|c|}
\hline \multirow{2}{*}{\multicolumn{2}{|c|}{ Segment }} & \multicolumn{2}{|c|}{ Starting Point (SP) } & \multirow{2}{*}{$\begin{array}{l}\text { SP Deviation } \\
/ \mathrm{m}\end{array}$} & \multirow{2}{*}{$\begin{array}{c}\text { Azimuth }(\mathrm{AB}, \mathrm{CD}) /^{\circ} \\
\text { Radius }(\mathrm{BC}) / \mathrm{m}\end{array}$} & \multirow{2}{*}{$\begin{array}{c}\text { Deviation } \\
\text { Azimuth }\left({ }^{\circ}\right) / \text { Radius }(\mathrm{m})\end{array}$} \\
\hline & & $\mathrm{X} / \mathrm{m}$ & $\mathrm{Y} / \mathrm{m}$ & & & \\
\hline \multirow{2}{*}{$\mathbf{A B}$ (Line) } & Design & -309.39 & -321.22 & \multirow{2}{*}{0.11} & 66.512 & \multirow{2}{*}{0.017} \\
\hline & Excavation & -309.42 & -321.33 & & 66.495 & \\
\hline \multirow{2}{*}{$\mathbf{B C}(\operatorname{Arc})$} & Design & 173.28 & -111.47 & \multirow{2}{*}{0.07} & 150.000 & \multirow{2}{*}{0.049} \\
\hline & Excavation & 173.23 & -111.42 & & 149.951 & \\
\hline \multirow{2}{*}{ CD (Line) } & Design & 263.49 & 25.59 & \multirow{2}{*}{0.11} & 0.190 & \multirow{2}{*}{0.002} \\
\hline & Excavation & 263.40 & 25.53 & & 0.192 & \\
\hline
\end{tabular}

Table 2. Results of the segment-wise centerline deviation evaluation.

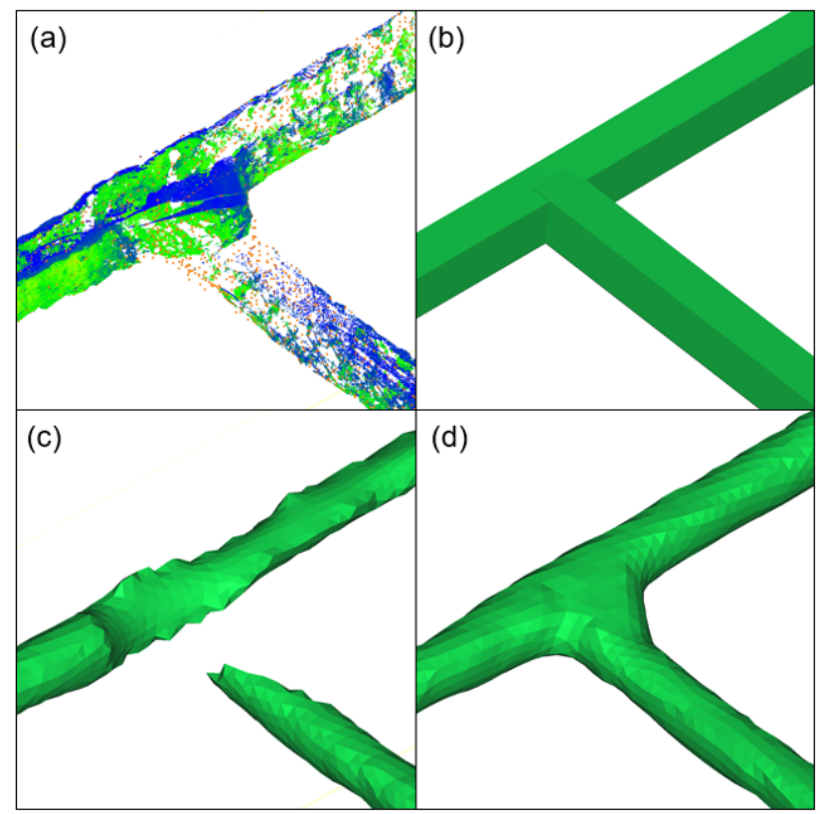

Figure 8. Comparison of point clouds and different models. (a) The collected TLS point clouds, the extracted and the completed featured points. Feature points are colored orange. (b) Design model; (c) reconstructed 3D model without feature point completion; (d) reconstructed 3D model after feature point completion.

due to their advantages in efficiency and data quality. Further studies are worthwhile using integrated laser scanning data from various platforms for tunnel inspection.

\section{CONCLUSION}

In this paper, a technical framework for evaluating tunnel excavation by incorporating TLS point clouds and design models is proposed. It firstly extracts feature points from each cross-section and then reduces the point deficiencies using the structure-aware point completion strategy towards an accurate 3D excavation model. Finally, the excavation quality is evaluated in terms of centerlines and $3 \mathrm{D}$ models. This work is validated in the case study. The results show that the centerline azimuth deviation is acceptable but considerable overbreak and underbreak need further drilling or backfilling.

\section{ACKNOWLEDGMENTS}

This work is jointly supported by the National Natural Science Foundation of China Projects (No. 41725005, No. 41901403).

\section{REFERENCES}

Attard, L., Debono, C. J., Valentino, G., Di Castro, M., 2018. Tunnel inspection using photogrammetric techniques and image processing: A review. ISPRS Journal of Photogrammetry and Remote Sensing, 144, 180-188.

Cui, H., Ren, X., Mao, Q., Hu, Q., Wang, W., 2019. Shield subway tunnel deformation detection based on mobile laser scanning. Automation in Construction, 106, 102889.

Dong, Z., Liang, F., Yang, B., Xu, Y., Zang, Y., Li, J., Wang, Y., Dai, W., Fan, H., Hyyppä, J. et al., 2020. Registration of largescale terrestrial laser scanner point clouds: A review and benchmark. ISPRS Journal of Photogrammetry and Remote Sensing, $163,327-342$.

Fekete, S., Diederichs, M., Lato, M., 2010. Geotechnical and operational applications for 3-dimensional laser scanning in drill and blast tunnels. Tunnelling and underground space technology, 25(5), 614-628.

Gikas, V., 2012. Three-dimensional laser scanning for geometry documentation and construction management of highway tunnels during excavation. Sensors, 12(8), 11249-11270.

Kang, Z., Zhang, L., Tuo, L., Wang, B., Chen, J., 2014. Continuous extraction of subway tunnel cross sections based on terrestrial point clouds. Remote Sensing, 6(1), 857-879.

Nurunnabi, A., Sadahiro, Y., Lindenbergh, R., 2017. Robust cylinder fitting in three-dimensional point cloud data. International Archives of the Photogrammetry, Remote Sensing and Spatial Information Sciences, 42(1/W1), 63-70.

Panella, F., Roecklinger, N., Vojnovic, L., Loo, Y., Boehm, J., 2020. Cost-benefit analysis of rail tunnel inspection for photogrammetry and laser scanning. International Archives of the Photogrammetry, Remote Sensing and Spatial Information Sciences-ISPRS Archives, ISPRS, 1137-1144.

Pejić, M., 2013. Design and optimisation of laser scanning for tunnels geometry inspection. Tunnelling and underground space technology, 37, 199-206.

Qiu, W., Cheng, Y.-J., 2017. High-resolution DEM generation of railway tunnel surface using terrestrial laser scanning data for clearance inspection. Journal of Computing in Civil Engineering, 31(1), 04016045.

Schnabel, R., Wahl, R., Klein, R., 2007. Efficient ransac for point-cloud shape detection. Computer graphics forum, 26, Wiley Online Library, 214-226. 
Soudarissanane, S., Lindenbergh, R., Menenti, M., Teunissen, P., 2011. Scanning geometry: Influencing factor on the quality of terrestrial laser scanning points. ISPRS journal of photogrammetry and remote sensing, 66(4), 389-399.

Wang, W., Zhao, W., Huang, L., Vimarlund, V., Wang, Z., 2014. Applications of terrestrial laser scanning for tunnels: a review. Journal of Traffic and Transportation Engineering (English Edition), 1(5), 325-337.

Xu, X., Yang, H., Neumann, I., 2018. A feature extraction method for deformation analysis of large-scale composite structures based on TLS measurement. Composite Structures, 184, 591-596.

Yi, C., Lu, D., Xie, Q., Liu, S., Li, H., Wei, M., Wang, J., 2019. Hierarchical tunnel modeling from 3D raw LiDAR point cloud. Computer-Aided Design, 114, 143-154. 